\title{
Bio-imaging the entry process of an emerging pathogenic virus: The West Nile virus.
}

\author{
JJH Chu, JWM Lee and ML Ng.
}

Flavivirology Laboratory, Department of Microbiology, 5 Science Dr. 2, National University of Singapore, Singapore 117597, Singapore.

\section{Introduction}

The intimate relationship between virus and host cells during the entry process is often a prerequisite for a successful infection. West Nile virus (WNV) is a mosquito-borne flavivirus belonging to the same family of dengue and Japanese encephalitis viruses. The entry of WNV into host cells is a complex and multi-steps process. Recently, WNV has been shown to bind its cellular receptor, $\alpha \mathrm{V} \beta 3$ integrin (Chu and $\mathrm{Ng}, 2004$ ) and domain III of WNV envelope protein is responsible for interacting with $\alpha \mathrm{V} \beta 3$ integrin (Chu et al., 2005). This critical interaction mediates the subsequent signaling pathway for the penetration of the virus into cells. In this study, the interacting forces between domain III of WNV envelope protein and $\alpha \mathrm{V} \beta 3$ integrin was analyzed using atomic force microscopy. In addition, the entry process of WNV was imaged live from the binding of virus to receptor, virus penetration and virus trafficking in relation to morphological changes in cells.

\section{Methods and Materials}

A combination of bio-imaging techniques, biochemical, molecular cloning and proteomics approaches was employed to decipher the entry process of WNV in detail. The bio-imaging techniques include the use of atomic force microscopy (AFM) to measure the engagement forces resulted from the protein-protein interaction between virus and cellular receptor. Laser scanning confocal microscopy was also used for the live imaging of virus entry process.

\section{Results and conclusions}

The AFM's metrological function was used to test and quantify the specificity and degree of binding interactions between WNV envelope protein domain III and integrin $\alpha \mathrm{V} \beta 3$ in a liquid environment. These proteins had been covalently bound to functionalized silicon nitride probes (NP-S) and silicon wafer substrates. The AFM measured the binding force between these 2 proteins as being $45 \pm 5 \mathrm{pN}$. Control experiments included the addition of integrin $\alpha \mathrm{V} \beta 3$ in solution to the system (which disrupted the binding between the WNV envelope protein domain III and integrin $\alpha \mathrm{V} \beta 3$ bound to the probe and wafer), as well as testing the interaction between WNV envelope protein domain III and integrin $\alpha \mathrm{V} \beta 5$ (which yielded no specific interactions).

Subsequent analysis of the WNV entry process after its engagement with $\alpha \mathrm{V} \beta 3$ integrin suggested the involvement of an integrin-associated signal induced clathrin-mediated 
endocytosis pathway. Co-localization of phosphorylated focal adhesion kinase with vinculin can be observed within 3 to 5 min post-infection of WNV. These processes further triggered the rearrangement of filamentous actin for the clathrin-mediated endocytosis of the virus particles. The rearrangement of actin filaments can be arrested by actin-disrupting drug (cytochalasin D) and cells treated with cytochalasin D are less susceptible to WNV infection. This showed the importance of F-actin in the initial endocytosis of WNV across the plasma membrane.

WNV also uses the host cell transport systems to achieve intracellular targeting of the incoming virion for uncoating and these were observed using the live-imaging capability of the confocal microscopy. Live tracking of endosomes and lysosomes in cells was achieved by labelling these organelles with Lysotracker Red DND-99 probe (Molecular Probes). The microtuble network was labeled with green fluorescent protein (GFP) by transfecting the cells with mammalian expression vector (pEGFP-Tub, Clonetech, USA) that expresses GFP-tagged tubulin monomers. WNV particles are labeled with Cy5 fluorescent dye. WNV particles enclosed within early endosomes are trafficked along the microtubules network from the cell periphery to the perinuclear region. The track taken by one of the endocytic vesicles with WNV was traced over a period of $120 \mathrm{sec}$. The distance travelled by a typical vesicle was measured to be approximately $12 \mu \mathrm{m}$ and the speed of its movement was determined to be $0.1 \mu \mathrm{m} / \mathrm{sec}$. The average speed of moving along the microtubule network derived from a total of 50 endocytic vesicles with WNV moving along the microtubules network was determined to be approximately $0.3 \mu \mathrm{m} / \mathrm{sec}$. This trafficking process can be disrupted with nacodazole (microtubule disrupting drug). This study demonstrated the importance of bio-imaging techniques in providing the necessary information that would provide the basis of anti-viral therapy that is directed towards the early phase of the WNV replication cycle.

\section{References}

(1) Chu JJ, Ng ML. (2004). Interaction of West Nile virus with $\alpha \mathrm{V} \beta 3$ integrin mediates virus entry into cells. J Biol Chem. 279(52): 54533-545341.

(2) Chu JJ, Rajamanonmani R, Li J, Bhuvanakantham R, Lescar J, Ng ML. (2005). Inhibition of West Nile virus entry by using a recombinant domain III from the envelope glycoprotein. J Gen Virol. 86: 405-412. 